Vol. XX (XXXX)

No. X

\title{
NONHOLONOMIC CONSTRAINTS IN CLASSICAL FIELD THEORIES
}

\author{
Ernst Binz \\ Fakultät für Mathematik und Informatik \\ Universität Mannheim, 68131 Mannheim, Germany \\ binz@math.uni-mannheim.de \\ Manuel de León and David Martín de Diego * \\ IMAFF, Consejo Superior de Investigaciones Científicas \\ Serrano 123, 28006 Madrid, Spain \\ mdeleon@imaff.cfmac.csic.es and d.martin@imaff.cfmac.csic.es \\ Dan Socolescu \\ Fachbereich Mathematik, Universität Kaiserslautern \\ 67663 Kaiserlautern, Germany \\ socolescu@mathematik.uni-kl.de \\ (Received \\ 2001)
}

\begin{abstract}
A multisymplectic setting for classical field theories subjected to non-holonomic constraints is presented. The infinite dimensional setting in the space of Cauchy data is also given.
\end{abstract}

Key words: non-holonomic constraints, Classical field theories, multisymplectic formalism.

\section{Introduction}

There is a renewal interest in two, in principle, different topics. One is the multisymplectic formalism for classical field theories, introduced in the latest seventies by the Polish school led by Tulczyjew (see [4] for details), and independently, by P.L. García et al 15, 16], and H. Goldsmichdt and Sh. Sternberg [17] (see [6, 7, 12, 13, 18, 19, 20, 21, 29] for recent approaches). The other topic is mechanical systems subjected to non-holonomic constraints. This last topic, being a classical subject in the literature, has recently become very prominent in the area of Geometric Mechanics.

Our aim in this paper is to give a multisymplectic setting for first-order field theories subjected to non-holonomic constraints, that is, the lagrangian depends on the first derivatives of the fields, and, in addition, not all the values are allowable. We do not

Partially supported by grants DGICYT (Spain) PB97-1257 and PGC2000-2191-E, and the University of Mannheim. 
know interesting examples in physics: for instance, $\operatorname{div} \mathbf{E}=0$ in electromagnetism, or $\operatorname{div} \mathbf{E}=\rho$ in electromagnetism interacting with charged matter (see [27]) are nonholonomic constraints, but they are dinamically invariant, so that nothing new can be added using our formalism. Continuum Mechanics seems to be the main and most interesting source of examples.

In 27 the authors consider incompressibility constraint in fluids and elastic solids, but this type of constraints become holonomic after integration in the space of Cauchy data. A genuine type of constraints are exhibited by an elastic body rolling with constant velocity, while deforming (for instance, an automobile tire on pavement [28]). Another important family of constraints are those appearing in the dynamics of media with microstructure. Let us recall that a simple body is mathematically modelled as a threedimensional manifold $B$ which can be covered by just one chart; therefore, a configuration is just an embedding of $B$ into $\mathbb{R}^{3}$, and a deformation is a change of configuration. A medium with microstructure is more involved: it is modelled as a principal bundle $P \longrightarrow B$ which is embedded in a physical ambient principal bundle. The configurations and the deformations are now principal bundle isomorphisms [2]. A multisymplectic setting for elastic media was developed in [22], and it is a natural continuation of the nice work in 26]. Many of these continuum systems exhibit kinematic constraints given by the existence of a connection in the ambient principal bundle [3, 9].

In this paper, apart from presenting a multisymplectic setting for first-order field theories subjected to non-holonomic constraints, we also develop the corresponding infinite dimensional setting in the space of Cauchy data, proving that the constraint forces can be integrated over the Cauchy surface to get a constrained 2-form on the space of Cauchy data. So, the equations of motion are obtained modifying the usual 2-form with this constrained 2-form.

\section{Multisymplectic geometry}

In this section we will recall some fundamentals on multisymplectic manifolds which will be needed later. We refer to [6] for more details.

Definition 1. A closed $k$-form $\omega$ on a manifold $M$ is called multisymplectic if the mapping

$$
X \in T_{x} M \mapsto i_{X} \omega \in\left(\Lambda^{k-1} E\right)_{x}
$$

is injective for all $x \in M$. In this case, the pair $(M, \omega)$ is called a multisymplectic manifold.

As is well-known, cotangent bundles are the canonical models for symplectic manifolds. For multisymplectic manifolds we have more variety of models (see [6]) but we are mainly interested in the following ones.

Let $\Lambda^{k} E$ be the total space of the bundle of $k$-forms on a manifold $E$. We define a $k$-form on $\Lambda^{k} E$ by setting

$$
\Theta(\alpha)\left(X_{1}, \ldots, X_{k}\right)=\alpha\left(T \rho_{E}^{k}(\alpha) X_{1}, \ldots, T \rho_{E}^{k}(\alpha) X_{k}\right),
$$


for $\alpha \in\left(\Lambda^{k} E\right)_{x}$ and $X_{1}, \ldots, X_{k} \in T_{\alpha}\left(\Lambda^{k} E\right)$, where $\rho_{E}^{k}: \Lambda^{k} E \longrightarrow E$ is the canonical projection.

The form $\Omega=-d \Theta$ is a multisymplectic $(k+1)$-form on $\Lambda^{k} E$. Of course, for $k=1$ we obtain the canonical symplectic form $\omega_{E}$ on $\Lambda^{1} E=T^{*} E$.

More interesting models can be defined if $E$ is fibered over a manifold $N$, say $\pi$ : $E \longrightarrow N$ is a fibration. Indeed, we consider the bundle of $r$-semibasic forms on $E$ :

$$
\Lambda_{r}^{k} E=\left\{\alpha \in \Lambda^{k} E \mid i_{v_{1} \wedge \cdots \wedge v_{r}} \alpha=0 \text {, for } r \pi \text { - vertical tangent vectors } v_{1}, \ldots, v_{r}\right\} .
$$

We can reproduce the above definition to obtain a $k$-form $\Theta_{r}$ on $\Lambda_{r}^{k} E$ which is nothing but the restriction of $\Theta$ to the submanifold $\Lambda_{r}^{k} E$. Therefore, the $(k+1)$-form $\Omega_{r}=-d \Theta_{r}$ is again multisymplectic and, of course, the restriction of $\Omega$.

\section{Multisymplectic formalism for classical field theories}

\subsection{The finite dimensional setting}

The mathematical ingredients of a classical field theory are the following ones:

- a fibration $\pi_{X Y}: Y \longrightarrow X$, where $X$ represents a $n$-dimensional space-time, that is, an oriented manifold of dimension $n$ with volume form $\eta$;

- a lagrangian form $\Lambda: Z \longrightarrow \Lambda^{n} X$, where $Z$ denotes the 1-jet prolongation $J^{1} \pi_{X Y}$.

There are two induced fibrations $\pi_{X Z}: Z \longrightarrow X$ and $\pi_{X Y}: Y \longrightarrow X$ such that $\pi_{X Z}=\pi_{X Y} \circ \pi_{Y Z}$.

We have $\Lambda=L \eta$, with the obvious identifications, and $L: Z \longrightarrow \mathbb{R}$ is the lagrangian function.

In this paper we will choose fibered coordinates $\left(x^{\mu}, y^{i}, z_{\mu}^{i}\right)$ on $Z$ such that $\eta=d^{n} x=$ $d x^{1} \wedge \cdots \wedge d x^{n}$. A useful notation will be $d^{n-1} x^{i}=i \frac{\partial}{\partial x^{i}} \eta$.

Therefore, the lagrangian function is $L=L\left(x^{\mu}, y^{i}, z_{\mu}^{i}\right)$. $L$ is said to be regular if the hessian matrix

$$
\left(\frac{\partial^{2} L}{\partial z_{\mu}^{i} \partial z_{\nu}^{j}}\right)
$$

is regular.

We define the action integral

$$
A(\sigma)=\int_{U}\left(j^{1} \sigma\right)^{*} \Lambda
$$

where $\sigma$ is a section of $\pi_{X Y}$ defined on an open set $U$, and $j^{1} \sigma$ denotes its first jet prolongation. A section $\sigma$ is called an extremal for the above action if

$$
\frac{d}{d t} A\left(\phi_{t} \circ \sigma\right)_{\mid t=0}=0
$$


for every flow $\phi_{t}$ on $Y$ such that $\pi_{X Y} \circ \phi_{t}=\pi_{X Y}$, and $\phi_{t}(y)=y$ for all $y$ in the boundary of $\sigma(U)$. Since such a flow $\phi_{t}$ is generated by a $\pi_{X Y}$-vertical vector field $\xi_{Y}$ vanishing on the boundary of $\sigma(U)$, we then conclude that $\sigma$ is an extremal if and only if

$$
\int_{U}\left(j^{1} \sigma\right)^{*} \mathcal{L}_{\xi_{Z}} \Lambda=0,
$$

for all $\xi_{Y}$ satisfying the above conditions, where $\xi_{Z}$ is the natural prolongation of $\xi_{Y}$ to $Z$. Let us recall that if

$$
\xi_{Y}=\xi^{i}(x, y) \frac{\partial}{\partial y^{i}},
$$

then

$$
\xi_{Z}=\xi^{i} \frac{\partial}{\partial y^{i}}+\frac{\partial \xi^{i}}{\partial x^{\mu}} \frac{\partial}{\partial z_{\mu}^{i}} .
$$

Therefore, we deduce that $\sigma\left(x^{\mu}\right)=\left(x^{\mu}, \sigma^{i}(x)\right)$ is an extremal if and only if

$$
\int_{U}\left[\frac{\partial L}{\partial y^{i}}-\frac{\partial}{\partial x^{\mu}}\left(\frac{\partial L}{\partial z_{\mu}^{i}}\right)\right] \xi^{i} d x^{n}=0
$$

for all values of $\xi^{i}$. Thus, $\sigma$ will be an extremal if and only if

$$
\frac{\partial L}{\partial y^{i}}-\frac{\partial}{\partial x^{\mu}}\left(\frac{\partial L}{\partial z_{\mu}^{i}}\right)=0
$$

along $j^{1} \sigma$. Equations (2) are called the Euler-Lagrange equations for $L$.

Next, we will recall the multisymplectic formulation of classical field theories.

First of all, using the volume form $\eta$ we can construct a tensor field of type $(1, n)$ on $Z$ :

$$
S_{\eta}=\left(d y^{i}-z_{\mu}^{i} d x^{\mu}\right) \wedge d^{n-1} x^{\nu} \otimes \frac{\partial}{\partial z_{\nu}^{i}} .
$$

The Poincaré-Cartan $n$ and $(n+1)$-forms are defined as

$$
\Theta_{L}=\Lambda+S_{\eta}^{*}(d L), \quad \Omega_{L}=-d \Theta_{L},
$$

where $S_{\eta}^{*}$ is the adjoint operator.

Proposition 1. The $(n+1)$-form $\Omega_{L}$ is multisymplectic if and only if the lagrangian $L$ is regular.

The integral action reads now as

$$
A(\sigma)=\int_{U}\left(j^{1} \sigma\right)^{*} \Theta_{L} .
$$

The use of the Poincaré-Cartan $(n+1)$-form allows us to characterize the extremals as follows. 
Proposition 2. [4] $\sigma$ is an extremal for the action if and only if

$$
\left(j^{1} \sigma\right)^{*}\left(i_{\xi_{Z}} \Omega_{L}\right)=0
$$

for all vector fields $\xi_{Z}$ on $Z$.

Eq. (3) gives the field equations, but we can consider a more general problem: to look for a section $\tau$ of the fibration $\pi_{X Z}: Z \longrightarrow X$ such that

$$
\tau^{*}\left(i_{\xi_{Z}} \Omega_{L}\right)=0
$$

for all vector fields $\xi_{Z}$ on $Z$. Eq. (4) is referred as the De Donder equations.

Looking the De Donder problem, one realizes that we search for sections $\tau$ of $\pi_{X Z}$ satisfying Eq. (住). We will linearize the problem as follows. First, notice that the subspace $H_{\tau(x)}=d \tau(x)\left(T_{x} X\right) \subset T_{\tau(x)} Z$ is horizontal, for every $x$ in the domain of $\tau$. Indeed, we have

$$
T_{\tau(x)} Z=H_{\tau(x)} \oplus\left(V \pi_{X Z}\right)_{\tau(x)},
$$

where $V \pi$ denotes the vertical bundle. Therefore, these horizontal subspaces can be considered as infinitesimal approximations to the section in the same way that tangent vectors are the linear approximations to the trajectories in particle mechanics.

Indeed, consider an arbitrary Ehresmann connection $\Gamma$ in the fibration $\pi_{X Z}: Z \longrightarrow$ $X$, that is, we have an horizontal distribution $H$ which is complementary of the vertical one:

$$
T Z=H \oplus V \pi_{X Z}
$$

Denote by $\mathbf{h}$ the horizontal projector $\mathbf{h}: T Z \longrightarrow H$. We then have

$$
\mathbf{h}\left(\frac{\partial}{\partial x^{\mu}}\right)=\frac{\partial}{\partial x^{\mu}}+\Gamma_{\mu}^{i} \frac{\partial}{\partial y^{i}}+\Gamma_{\nu \mu}^{i} \frac{\partial}{\partial z_{\nu}^{i}} .
$$

A direct computation shows that

$$
i_{\mathbf{h}} \Omega_{L}=(n-1) \Omega_{L}
$$

if and only if

$$
\begin{array}{r}
\left(\Gamma_{\nu}^{j}-z_{\nu}^{j}\right)\left(\frac{\partial^{2} L}{\partial z_{\mu}^{i} \partial z_{\nu}^{j}}\right)=0 \\
\frac{\partial L}{\partial y^{i}}-\frac{\partial^{2} L}{\partial x^{\mu} \partial z_{\mu}^{i}}-\Gamma_{\mu}^{j} \frac{\partial^{2} L}{\partial y^{j} \partial z_{\mu}^{i}}-\Gamma_{\mu \nu}^{j} \frac{\partial^{2} L}{\partial z_{\mu}^{j} \partial z_{\nu}^{i}}+\left(\Gamma_{\nu}^{j}-z_{\nu}^{j}\right) \frac{\partial^{2} L}{\partial y^{i} \partial z_{\nu}^{j}}=0
\end{array}
$$

(see 23]).

If the lagrangian $L$ is regular, then Eq. (6) implies that $\Gamma_{\mu}^{i}=z_{\mu}^{i}$ and therefore (17) becomes

$$
\frac{\partial L}{\partial y^{i}}-\frac{\partial^{2} L}{\partial x^{\mu} \partial z_{\mu}^{i}}-z_{\mu}^{j} \frac{\partial^{2} L}{\partial y^{j} \partial z_{\mu}^{i}}-\Gamma_{\mu \nu}^{j} \frac{\partial^{2} L}{\partial z_{\mu}^{j} \partial z_{\nu}^{i}}=0
$$


Now, if $\tau\left(x^{\mu}\right)=\left(x^{\mu}, \tau^{i}(x), \tau_{\mu}^{i}(x)\right)$ is an integral section of $H$ we would have

$$
z_{\mu}^{i}=\frac{\partial \tau^{i}}{\partial x^{\mu}}, \Gamma_{\mu \nu}^{i}=\frac{\partial \tau_{\mu}^{i}}{\partial x^{\nu}}
$$

which proves that Eq. (8) is nothing but the Euler-Lagrange equations for $L$.

As we have noticed, for regular lagrangians the field and De Donder equations are equivalent, that is, a solution $\tau$ of the De Donder problem is automatically a jet prolongation of a solution of the field equations.

Eq. (5) can be considered as the linear version of De Donder equations. If the lagrangian $L$ is regular, a global solution always exists, because one can choose local solutions and use then a partition of the unity to glue together all the local solutions. But this solution will not be unique. Indeed, given a particular solution $\Gamma$ then any other solution is of the form $\mathbf{h}+T$, where $T=T_{\mu \nu}^{i} d x^{\nu} \otimes \frac{\partial}{\partial z_{\mu}^{i}}$ is a tensor field on $Z$ of type $(1, n)$ satisfying the following conditions

$$
T_{\mu \nu}^{i} \frac{\partial^{2} L}{\partial z_{\mu}^{i} \partial z_{\nu}^{j}}=0, \text { for all } j .
$$

\subsection{Presymplectic formalism in the space of Cauchy data}

Next, we will show how the selection of a Cauchy surface permits to give an infinite dimensional setting for field theories (see [4] for a detailed exposition).

A Cauchy surface is a compact oriented $(n-1)$-dimensional submanifold $B$ of $X$. We shall consider a manifold of embeddings from $B$ into $X$, denoted by $\tilde{X}$; it will be the space of parametrized Cauchy surfaces.

A space of Cauchy data will be a manifold $\tilde{Z}$ of embeddings from $B$ into $Z$ such that

$\pi_{X Z} \circ \gamma \in \tilde{X}$, for all embedding $\gamma \in \tilde{Z}$. This condition permits to define a fibration $\pi_{\tilde{X} \tilde{Z}}: \tilde{Z} \longrightarrow \tilde{X}$.

A space of Dirichlet data is a manifold $\tilde{Y}$ of embeddings from $B$ to $Y$ such that $\pi_{Y Z} \circ \gamma \in \tilde{Y}$, for all $\gamma \in \tilde{Z}$. Therefore we have a fibration $\pi_{\tilde{Y} \tilde{Z}}: \tilde{Z} \longrightarrow \tilde{Y}$ such that $\pi_{\tilde{X} \tilde{Z}}=\pi_{\tilde{X} \tilde{Y}} \circ \pi_{\tilde{Y} \tilde{Z}}$.

We define a 1 -form $\tilde{\Theta}$ on $\tilde{Z}$ as follows:

$$
\tilde{\Theta}(\gamma)(\xi)=\int_{B} \gamma^{*}\left(i_{\xi} \Theta_{L}\right)
$$

where $\gamma \in \tilde{Z}$, and $\xi \in T_{\gamma} \tilde{Z}$. Notice that such a tangent vector $\xi$ can be viewed as a mapping $\xi: B \longrightarrow T Z$ covering $\gamma$.

Proposition 3. [4] We have

$$
d \tilde{\Theta}(\gamma)\left(\xi_{1}, \xi_{2}\right)=-\int_{B} \gamma^{*}\left(i_{\xi_{2}} i_{\xi_{1}} \Omega_{L}\right)
$$

for all tangent vectors $\xi_{1}, \xi_{2} \in T_{\gamma} \tilde{Z}$ and for all $\gamma \in \tilde{Z}$. 
Let $c_{\tilde{X}}(t)$ be a curve in $\tilde{X}$. We say that $c_{\tilde{X}}(t)$ splits $X$ if the mapping

$$
(b, t) \mapsto c_{\tilde{X}}(t)(b)
$$

is a diffeomorphism from $B \times \mathbb{R}$ into $X$. (For simplicity, we are assuming that the curves are defined in the whole real line).

Let now $c_{\tilde{Z}}(t)$ be a curve in $\tilde{Z}$ such that its projection $\pi_{\tilde{X} \tilde{Z}} \circ c_{\tilde{Z}}(t)$ splits $X$. Under this condition, one can define a section $\tau: X \longrightarrow Z$ of $\pi_{X Z}$ as follows:

$$
\tau(x)=c_{\tilde{Z}}(t)(b)
$$

since $x=\pi_{X Z} \circ c_{\tilde{Z}}(t)(b)$.

Conversely, given a section $\tau$ of $\pi_{X Y}$ and a curve $c_{\tilde{X}}(t)$ in $\tilde{X}$, we define a curve $c_{\tilde{Z}}(t)$ in $\tilde{Z}$ by the formula

$$
c_{\tilde{Z}}(t)(b)=\tau\left(c_{\tilde{X}}(t)(b)\right) .
$$

Theorem 1. Wet $\tau$ be a section of $\pi_{X Z}$, and $c_{\tilde{X}}(t)$ a curve in $\tilde{X}$ such that $c_{\tilde{X}}(t)(B)$ is contained in the domain of $\tau$. Let $c_{\tilde{Z}}(t)$ be the lift of $c_{\tilde{X}}(t)$ to $\tilde{Z}$ as we described above. If $\tau$ satisfies the De Donder equation then

$$
i_{\dot{c}_{\tilde{Z}}(t)} d \tilde{\Theta}=0 .
$$

Conversely, if the curve $c_{\tilde{Z}}(t)$ satisfies $E q$. (9) and its projection splits $X$, then the section $\tau$ constructed as above satisfies the De Donder equations.

\section{Classical field theories with non-holonomic constraints}

A non-holonomic classical field theory consists of a lagrangian function $L=L\left(x^{\mu}, y^{i}, z_{\mu}^{i}\right)$ subjected to constraints of the form

$$
\Phi^{\alpha}\left(x^{\mu}, y^{i}, z_{\mu}^{i}\right)=0,1 \leq \alpha \leq k .
$$

This means that the solutions of the field equations have to satisfy the above equations along the full evolution of the system.

Definition 2. A section $\sigma$ of $\pi_{X Y}: Y \longrightarrow X$ is a solution of the nonholonomic problem if (1) holds for all vector fields $\xi_{Y}$ on $Y$ satisfying the condition

$$
\frac{\partial \Phi^{\alpha}}{\partial z_{\mu}^{i}} \xi^{i}=0, \quad \alpha=1, \ldots, k .
$$

REMARK 1. The above definition could be considered as a sort of d'Alembert principle for constrained field theories. In principle, we consider non-linear constraints. At the end of this section we will treat the particular case of linear or affine constraints. 
Proceeding as in the case of unconstrained systems we deduce that $\sigma$ is a solution of the nonholonomic problem if and only if

$$
\int_{U}\left[\frac{\partial L}{\partial y^{i}}-\frac{\partial}{\partial x^{\mu}}\left(\frac{\partial L}{\partial z_{\mu}^{i}}\right)\right] \xi^{i} d x^{n}=0
$$

for all values of $\xi^{i}$ satisfying (10) (here $U$ is the domain of definition of $\sigma$ ).

Therefore, such a solution $\sigma\left(x^{\mu}\right)=\left(x^{\mu}, \sigma^{i}(x)\right)$ would satisfy the following set of equations:

$$
\begin{aligned}
\frac{\partial L}{\partial y^{i}}-\frac{\partial}{\partial x^{\mu}}\left(\frac{\partial L}{\partial z_{\mu}^{i}}\right) & =\lambda_{\alpha \mu} \frac{\partial \Phi^{\alpha}}{\partial z_{\mu}^{i}} \\
\Phi^{\alpha}\left(x^{\mu}, \sigma^{i}, \frac{\partial \sigma^{i}}{\partial x^{\mu}}\right) & =0
\end{aligned}
$$

along $j^{1} \sigma$. Equations (11) are called the non-holonomic field equations for $L$. Here, the $\lambda_{\alpha \mu}$ are some Lagrange multipliers to be obtained.

We will rewrite the non-holonomic problem in a geometrical manner.

First of all, the constraints geometrically define a submanifold $M$ of $Z$. Therefore, a field theory with non-holonomic constraints is described by a lagrangian form $\Lambda=L \eta$ and a submanifold $M$ of $Z$.

The non-holonomic De Donder equations can be then written as follows:

$$
i_{\mathbf{h}} \Omega_{L}-(n-1) \Omega_{L} \in \mathcal{Y}\left(S_{\eta}^{*}\left(T M^{o}\right)\right), \quad \operatorname{Im} \mathbf{h} \subset T M
$$

where $\mathbf{h}$ is the horizontal projector of a Ehresmann connection $\Gamma$ in $\pi_{X Z}: Z \longrightarrow X, T M^{o}$ is the annihilator of $T M$, and $\mathcal{Y}\left(S_{\eta}^{*}\left(T M^{o}\right)\right)$ denotes the ideal generated by the space of $n$-forms $S_{\eta}^{*}\left(T M^{o}\right)$.

We will check that, in fact, the above equations are correct. Indeed, the first equation in (13) can be written as

$$
i_{\mathbf{h}} \Omega_{L}-(n-1) \Omega_{L}=\lambda_{\alpha} \wedge S_{\eta}^{*}\left(d \Phi^{\alpha}\right),
$$

with some 1-forms $\lambda_{\alpha}$.

In the following, we will assume the following condition:

\section{Assumption on the regularity of the constraints:}

The $n$-forms

$$
\left\{S_{\eta}^{*}\left(d \Phi^{\alpha}\right) \mid \alpha=1, \ldots, k\right\}
$$

are linearly independent.

Under this regularity assumption, it is esay to see that

$$
\lambda_{\alpha}=\lambda_{\alpha \mu} d x^{\mu} .
$$


Thus, if we introduce the notation

$$
\theta_{\mu}=\lambda_{\alpha \mu} S_{\eta}^{*}\left(d \Phi^{\alpha}\right)
$$

we deduce that (14) can be written as follows:

$$
i_{\mathbf{h}} \Omega_{L}-(n-1) \Omega_{L}=d x^{\mu} \wedge \theta_{\mu} .
$$

Now, from (13) we obtain

$$
\begin{aligned}
&\left(\Gamma_{\nu}^{j}-z_{\nu}^{j}\right)\left(\frac{\partial^{2} L}{\partial z_{\mu}^{i} \partial z_{\nu}^{j}}\right)=0 \\
& \frac{\partial L}{\partial y^{i}}-\frac{\partial^{2} L}{\partial x^{\mu} \partial z_{\mu}^{i}}-\Gamma_{\mu}^{j} \frac{\partial^{2} L}{\partial y^{j} \partial z_{\mu}^{i}}-\Gamma_{\mu \nu}^{j} \frac{\partial^{2} L}{\partial z_{\mu}^{j} \partial z_{\nu}^{i}}+\left(\Gamma_{\nu}^{j}-z_{\nu}^{j}\right) \frac{\partial^{2} L}{\partial y^{i} \partial z_{\nu}^{j}}=\lambda_{\alpha \mu} \frac{\partial \Phi^{\alpha}}{\partial z_{\mu}^{i}}
\end{aligned}
$$

If the lagrangian $L$ is regular, we deduce from (16) that $\Gamma_{\mu}^{j}=z_{\mu}^{j}$, and consequently, (17) becomes

$$
\frac{\partial L}{\partial y^{i}}-\frac{\partial}{\partial x^{\mu}}\left(\frac{\partial L}{\partial z_{\mu}^{i}}\right)=\lambda_{\alpha \mu} \frac{\partial \Phi^{\alpha}}{\partial z_{\mu}^{i}}
$$

If $\tau\left(x^{\mu}\right)=\left(x^{\mu}, \tau^{i}(x), \tau_{\mu}^{i}(x)\right)$ is an integral section of $H$ we would have

$$
z_{\mu}^{i}=\frac{\partial \tau^{i}}{\partial x^{\mu}}, \quad \Gamma_{\mu \nu}^{i}=\frac{\partial \tau_{\mu}^{i}}{\partial x^{\nu}}
$$

and thus Eq. (18) gives the non-holonomic field equations for $L$.

Next we will give the infinite dimensional setting for non-holonomic field theory.

To do that, we prove the following result.

Proposition 4. Let $\mathbf{h}$ be the horizontal projector of an Ehresmann connection in the fibration $\pi_{X Z}: Z \longrightarrow X$, and let $\tau: X \longrightarrow Z$ be an integral section of this connection. If $\mathbf{h}$ is a solution of the non-holonomic De Donder equations, then we have

$$
\tau^{*}\left(i_{\xi_{Z}}\left(\Omega_{L}-d x^{\mu} \wedge \theta_{\mu}\right)\right)=0,
$$

for all vector fields $\xi_{Z}$ on $Z$. Conversely, if for all the integral sections $\tau$ of $\mathbf{h} E q$. (19) holds then $\mathbf{h}$ is a solution of the non-holonomic De Donder equations.

Eq. (19) can be viewed as the non-holonomic version of the De Donder problem.

Choose now a Cauchy surface $B$ and the corresponding spaces $\tilde{X}, \tilde{Y}$ and $\tilde{Z}$. It should be noticed that now the space of Cauchy data $\tilde{Z}$ consists of embeddings $\gamma: B \longrightarrow Z$ taking values in $M$.

As above we integrate the Poincaré-Cartan $(n+1)$-form $\Omega_{L}$ to construct a 2 -form $d \tilde{\Theta}$. We will proceed in the same way with the constraints. Let $\Xi$ be the $(n+1)$-form on $Z$ defined by

$$
\Xi=d x^{\mu} \wedge \theta_{\mu} .
$$


We then define a 2-form $\tilde{\Xi}$ on $\tilde{Z}$ as follows:

$$
\tilde{\Xi}(\gamma)\left(\xi_{1}, \xi_{2}\right)=-\int_{B} \gamma^{*}\left(i_{\xi_{2}} i_{\xi_{1}} \Xi\right),
$$

for all $\gamma \in \tilde{Z}$ and for all tangent vectors $\xi_{1}, \xi_{2} \in T_{\gamma} \tilde{Z}$.

TheOrem 2. Let $\tau$ be a section of $\pi_{X Z}$ taking values in $M$, and $c_{\tilde{X}}(t)$ a curve in $\tilde{X}$ such that $c_{\tilde{X}}(t)(B)$ is contained in the domain of $\tau$. Let $c_{\tilde{Z}}(t)$ be the lift of $c_{\tilde{X}}(t)$ to $\tilde{Z}$ as we described in the preceding section. If $\tau$ satisfies the non-holonomic De Donder equation (19) then

$$
i_{\dot{c}_{\tilde{Z}}(t)}(d \tilde{\Theta}-\tilde{\Xi})=0 .
$$

Conversely, if the curve $c_{\tilde{Z}}(t)$ satisfies Eq. (20) and its projection splits $X$, then the section $\tau$ constructed as above satisfies the non-holonomic De Donder equations.

Proof: The proof follows the same lines that in [⿰] if $\tau$ satisfies (19), then we have

$$
\begin{aligned}
\left(i_{\dot{c}_{\tilde{Z}}(t)} d \Theta\right)\left(\xi_{2}\right) & =-\int_{B} c_{\tilde{Z}}(t)^{*}\left(i_{\xi_{2}} i_{\dot{c}_{\tilde{Z}}(t)} \Omega_{L}\right) \\
& =-\int_{B} c_{\tilde{Z}}(t)^{*}\left(i_{\xi_{2}} i_{\dot{c}_{\tilde{Z}}(t)} \Xi\right) \\
& =\tilde{\Xi}\left(c_{\tilde{Z}}(t)\right)\left(\dot{c}_{\tilde{Z}}(t), \xi_{2}\right) \\
& =\left(i_{\dot{c}_{\tilde{Z}}}(t) \tilde{\Xi}\right)\left(\xi_{2}\right)
\end{aligned}
$$

which implies (20).

The converse is proved by reversing the argument.

\section{Affine and linear constraints}

Let $\mathcal{E}$ be a subspace of $\Lambda_{2}^{n} Y$. As we know, an element $z=j_{x}^{1} \sigma \in Z$ can be viewed as an injective linear mapping $z=d \sigma(x): T_{x} X \longrightarrow T_{\sigma(x)} Y$. Therefore, we could define a submanifold $M$ of $Z$ as follows:

$$
M=\left\{z \in Z \mid d \sigma(x)\left(T_{x} X\right) \text { is annihilated by } \mathcal{E}\right\} .
$$

We will prove that $M$ is the zero set of a family of affine constraints.

Indeed, take a basis $\left\{\varphi^{\alpha}, \alpha=1, \ldots, k\right\}$ of $\mathcal{E}$, and notice that each element $\varphi^{\alpha}$ is locally expressed as

$$
\varphi^{\alpha}=\left(\varphi^{\alpha}\right)_{0} d^{n} x+\left(\varphi^{\alpha}\right)_{i}^{\mu} d y^{i} \wedge d^{n-1} x^{\mu} .
$$

Since

$$
d \sigma(x)\left(\frac{\partial}{\partial x^{\mu}}\right)=\frac{\partial}{\partial x^{\mu}}+z_{\mu}^{i} \frac{\partial}{\partial y^{i}},
$$


where $z=\left(x^{\mu}, y^{i}, z_{\mu}^{i}\right)$, we have

$$
\varphi^{\alpha}\left(d \sigma(x) \frac{\partial}{\partial x^{1}}, \ldots, d \sigma(x) \frac{\partial}{\partial x^{n}}\right)=\left(\varphi^{\alpha}\right)_{0}+\left(\varphi^{\alpha}\right)_{i}^{\mu} z_{\mu}^{i},
$$

which is an affine constraint. Linear constraints are obtained considering a subspace of the quotient vector bundle $Z^{*}=\frac{\Lambda_{2}^{n} Y}{\Lambda_{1}^{n} Y}$.

\section{An example: a pneumatic tire}

We shall briefly describe a mathematical model for an automobile tire rolling with constant velocity, while deforming.

There are several mathematical models: Rocard's theory, Greidanu's theory and Keldys's theory (see [28], Ch. VI, pp. 308 and ff.) The last one is the most interesting. We shall describe it.

For a pneumatic tire, we introduce the following parameters describing a configuration: the coordinate $x$ of the point $K$ of intersection of the diameter of greatest slope of the wheel and the plane $O x y$ of the road, the angle $\kappa$ between the normal to the road and the central plane of the wheel, the angle $\theta$ between the $O y$ axis and the path of the central plane of the wheel on the road, the lateral displacement $\xi$ of the center of the area of contact of the tire with respect to $K$, and the angle $\varphi$ measuring the torsional deformation of the tire (see Figure).

The lagrangian is

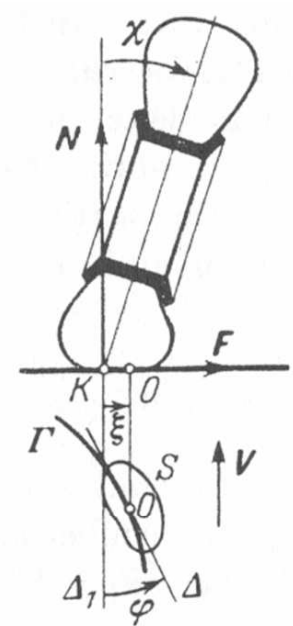

$$
L(x, \kappa, \theta, \xi, \varphi, \dot{x}, \dot{\kappa}, \dot{\theta}, \dot{\xi}, \dot{\varphi})=T(x, \kappa, \theta, \dot{x}, \dot{\kappa}, \dot{\theta})-U(\kappa, \xi, \varphi),
$$

where $T$ is the kinetic energy and $U$ is an elastic potential. The kinetic energy of deformation of the tire is neglected, and the potential energy is

$$
U(\xi, \phi, \kappa)=\frac{1}{2}\left(a \xi^{2}+b \phi^{2}+\rho N \kappa^{2}+2 \sigma N \xi \kappa\right),
$$

where $a, b, \sigma$, and $\rho$ are constants to be experimentally determined, and $N$ is the normal load. It should be noticed that when $N$ increases the area of contact of the tire, which remains at rest during the loading of the wheel, increases.

The kinematic constraints are

$$
\begin{aligned}
\dot{x}+\dot{\xi}+V \theta+V \phi & =0 \\
\dot{\theta}+\dot{\varphi}-\alpha V \xi+\beta V \varphi+\gamma V \kappa & =0,
\end{aligned}
$$

where $\alpha, \beta$ and $\gamma$ are constants, and $V$ is the constant velocity

of the wheel.

The stability of vehicles on wheels with pneumatic tires is carefully analyzed in [28], introducing several simplifications to treat with the motion equations. 
It should also be noticed that for accelerated motions, the area of the contact of the tire with road is not more constant (or even simply connected). This would lead to introduce unilateral constraints. There are mathematical descriptions for particle mechanic systems subjected to unilateral constraints (see 10, 11, 5] and the references therein) but the extension to classical field theories is not developed yet.

\section{Concluding remarks and future work}

We have developed a multisymplectic setting for first-order field theories subjected to non-holonomic constraints which is very similar to that for particle mechanics (see [24]). The dynamics in the corresponding space of Cauchy data was also analyzed.

\subsection{Algebraic computation of the Lagrange multipliers}

In Particle Mechanics the Lagrange multipliers are obtained using a simple algebraic method, indeed, the problem is reduced to apply the Cramer rule for systems of linear equations [24]. Therefore, one can use Mathematica ${ }^{\circledR}$ to do the computation. A similar procedure can be used for field theories, but the situation is really more complicated. In addition, we are trying to obtain the constrained Ehresmann connection by projecting a solution of the unconstrained problem.

\subsection{Applications to Continuum Mechanics}

As we said in the Introduction, the most interesting situations occur in Continuun Mechanics. The case of non-holonomic constraints given by connections in media with microstructure are being analyzed in [1]. Some independent work can be found in [14, 30].

\subsection{Non-holonomic bracket, non-holonomic momentum mapping and re- duction}

These topics will be analyzed in [1] and [25], following the lines in [7] and [8].

\section{Acknowledgements}

The authors wish to thank the referee for his comments and remarks that help to improve this paper.

\section{REFERENCES}

[1] E. Binz, M. de León, D. Martín de Diego and D. Socolescu: Multisymplectic formalism for classical field theories subjected to non-holonomic constraints, in preparation.

[2] E. Binz, M. de León and D. Socolescu: C.R. Acad. Sc. Paris, 326, Série II b, 227-232 (1998).

[3] E. Binz, M. de León and D. Socolescu: Extracta Mathematicae 14 2, 99-125 (1999).

[4] E. Binz, J. Sniatycki and H. Fischer: Geometry of classical fields, North-Holland, Amsterdam, 1988. 
[5] B. Brogliato: Non-smooth impact dynamics, Springer-Verlag, Berlin, 1996.

[6] F. Cantrijn, A. Ibort and M. de León: J. Austral. Math. Soc. (Series A) 66, 303-330 (1999).

[7] F. Cantrijn, A. Ibort and M. de León: Rend. Sem. Mat. Univ. Pol. Torino, 54, 3, 225-236 (1996).

[8] F. Cantrijn, M. de León, J.C. Marrero and D. Martín de Diego: J. Math. Phys. 40 , 795-820 (1999).

[9] G. Capriz: Continua with Microstructure, Springer Tracts in Natural Philosophy, 35, Springer, Berlin (1989).

[10] A. Ibort, E. Lacomba, M. de León, J.C. Marrero, D.M. de Diego and P. Pitanga: J. Phys. A: Math. Gen. 34, 1691-1712 (2001).

[11] J. Cortés, M. de León, D. Martín de Diego and S. Martínez: Royal Society of London Proceedings Series A: Math. Phys. Eng. Sci. 457 2007, 651-670 (2001).

[12] A. Echevarría-Enríquez, M.C. Muñoz-Lecanda and N. Román-Roy: J. Math. Phys. 39, 4578-4603 (1998).

[13] A. Echevarría-Enríquez, M.C. Muñoz-Lecanda and N. Román-Roy: J. Phys. A 32, 84618484 (1999).

[14] A. Fernández, P.L. García and C. Rodrigo: Lagrangian reduction and constrained variational calculus, in Proceedings of the IX Fall Workshop of Geometry and Physics, X. Grácia, M. Muñoz-Lecanda and N. Román-Roy eds., Publicaciones de la RSME vol. 3, Madrid, 2001.

[15] P.L. García and A. Pérez-Rendón: Comm. Math. Phys., 13 24-44 (1969).

[16] P.L. García and A. Pérez-Rendón: Arch. Rat. Mech. Anal. 43, 101-124 (1971).

[17] H. Goldschmidt and Sh. Sternberg: Ann. Inst. Fourier (Grenoble 23, 203-267 (1975).

[18] M.J. Gotay, J. Isenberg and J.E. Marsden: Momentun maps and classical relativistic fields, Part I: Covariant field theory, MSRI preprint (1997).

[19] M.J. Gotay, J. Isenberg and J.E. Marsden: Momentun maps and classical relativistic fields, Part II: Canonical analysis of field theories, MSRI preprint (1999).

[20] S.P.Hrabak: On a Multisymplectic Formulation of the Classical BRST symmetry for First Order Field Theories Part I: Algebraic Structures, math-ph/9901012 (1999).

[21] S.P.Hrabak: On a Multisymplectic Formulation of the Classical BRST Symmetry for First Order Field Theories Part II: Geometric Structures, math-ph/9901013 (1999).

[22] M. de León: Media with microstructure: constitutive theory and dynamics, in Proceedings of the IX Fall Workshop of Geometry and Physics, X. Grácia, M. Muñoz-Lecanda and N. Román-Roy eds., Publicaciones de la RSME vol. 3, Madrid, 2001.

[23] M. de León, J.C. Marrero and J. Marín: A geometrical approach to classical field theories: a constraint algorithm for singular theories, in New Developments in Differential Geometry (Debrecen, 1994), Math. Appl. 350, Kluwer, Dordrecht, 1996, pp. 291-312.

[24] M. de León and D. Martín de Diego: J. Math. Phys. 37, 3389-3414 (1996).

[25] M. de León, D. Martín de Diego and A. Santamaría: Symmetries and reduction in classical field theories, in preparation. 
[26] J.E. Marsden and Th.J.R. Hughes: Mathematical foundations of elasticity, Dover Publications, Inc., New York, (1994).

[27] J.E. Marsden, S. Pekarsky, S. Shkoller and M. West: J. Geom. Phys. 38, 253-284 (2001).

[28] Ju.I. Neimark, N.A. Fufaev: Dynamics of Nonholonomic systems, Translations of Mathematical Monoghraphs, 33, AMS, Providence, R.I, (1972).

[29] C. Paufler and H. Roemer: De Donder-Weyl Equations and Multisymplectic Geometry, to appear in Rep. Math. Phys. (2001).

[30] S. Vignolo and D. Bruno: Iper-ideal kinetic constraints in Continuum Mechanics, preprint 2001. 